\title{
BMJ Open Proton therapy for craniopharyngioma in adults: a protocol for systematic review and meta-analysis
}

\author{
Pengtao Li, ${ }^{1}$ Jialing Wang, ${ }^{2}$ Aximujiang Axier, ${ }^{1}$ Kai Zhou, ${ }^{1}$ Jingwei Yun, ${ }^{1}$ \\ Huayi Wang, ${ }^{1}$ Tingrong Zhang (D) , ${ }^{1}$ Shaoshan Li (D) ${ }^{1}$
}

To cite: Li P, Wang J, Axier A, et al. Proton therapy for craniopharyngioma in adults: a protocol for systematic review and meta-analysis. BMJ Open 2021;11:e046043. doi:10.1136/ bmjopen-2020-046043

- Prepublication history for this paper is available online To view these files, please visit the journal online (http://dx.doi org/10.1136/bmjopen-2020 046043).

PL and JW contributed equally.

Received 19 0ctober 2020 Accepted 19 May 2021

Dheck for updates

(C) Author(s) (or their employer(s)) 2021. Re-use permitted under CC BY-NC. No commercial re-use. See rights and permissions. Published by BMJ.

${ }^{1}$ Department of Neurosurgery, First Affiliated Hospital of Xinjiang Medical University, Urumqi, China

${ }^{2}$ Department of Anesthesiology, First Affiliated Hospital of Xinjiang Medical University, Urumqi, China

Correspondence to

Dr Shaoshan Li;

shaoshanli2019@163.com

\section{ABSTRACT}

Introduction Craniopharyngioma is the most challenging to treat brain tumour with high recurrence rates, which can be effectively reduced by adjuvant radiotherapy. In recent years, proton therapy (PT), with its physical properties of heavy ion beam, that is, Prague peak phenomenon, has been more frequently used in patients with craniopharyngioma. Compared with conventional X-ray beam radiotherapy, PT can reduce the damage to normal tissues and enlarge the damage to tumours. Some studies have shown that PT has advantages in the treatment of craniopharyngioma in adults. However, the optimal management of craniopharyngioma remains controversial. The purpose of this study was to evaluate the efficacy and safety of PT for craniopharyngioma in adults.

Methods and analysis We will search six databases (MEDLINE, EMBASE, Web of Science, the Cochrane Library, Amed, Scopus), clinical research registration websites and grey literature, aiming to identify randomised controlled trials (RCTs) on PT for craniopharyngioma in adults between 1 January 1954 and 28 September 2021. In the RCTs, PT will be used as the intervention group, and conventional $\mathrm{X}$-ray beam radiotherapy will be used as the comparator group. Tumour recurrence and survival will be the primary outcome, and treatment-related toxicity will be the secondary outcome. The study selection, data extraction, bias risk and quality evaluation will be operated by two to four researchers independently. We will use Review Manager V.5.2 (RevMan V.5.2) for data analysis. If there is significant heterogeneity, we will identify the source of heterogeneity by subgroup analysis.

Ethics and dissemination Our study is based on existing RCTs and does not require ethical approval. The results of the study will be published in a peer-reviewed journal or at a related conference.

PROSPERO registration number CRD42020200909.

\section{INTRODUCTION}

Craniopharyngioma is a benign tumour located in the sellar and parasellar regions of the brain. Its limited invasiveness and its proximity to the hypothalamus, pituitary, optic nerve and carotid artery make it the most challenging tumour in neurosurgery. The incidence of craniopharyngioma is 1.3 parts per million, which can occur in all age groups, showing a bimodal age distribution

\section{Strengths and limitations of this study}

First meta-analysis of which we are aware of the safety and efficacy of proton therapy for craniopharyngioma in adults.

- The results of this meta-analysis will provide an important reference for the treatment of craniopharyngioma in adults.

- Only randomised controlled trials will be included in this study.

- We will perform subgroup analysis according to different craniopharyngioma subtypes, locations and tumour sizes.

- Craniopharyngioma is a rare disease, which may face the problem of insufficient sample size.

(5-14 and 50-74 years old). ${ }^{1}$ However, the attention of craniopharyngioma in adults is far less than that in children. Surgical treatment of craniopharyngioma is difficult, and the rate of disability and mortality after gross total resection (GTR) is very high, but the recurrence rate of subtotal resection (STR) was $50 \%-91 \% .^{2-4}$ In recent years, more and more evidence shows that postoperative adjuvant radiotherapy (RT) for STR can achieve the same tumour control rate as GTR. ${ }^{5-8}$ A recent the Surveillance, Epidemiology, and End Results (SEER) analysis including 1218 patients with craniopharyngioma also demonstrated no significant difference in survival between RT, GTR and STR+RT. ${ }^{9}$ Conventional X-ray beam RT is difficult to achieve enough doses, and is prone to serious radiation-related toxicity, such as endocrine disorders, cognitive disorders, optic nerve injury and development of the second type of cancer. ${ }^{10-12}$

With the development of RT technology, proton therapy (PT) has been increasingly used in the treatment of craniopharyngioma. ${ }^{13-17}$ The physical properties of proton beam and Bragg peak phenomenon can reduce the damage to normal 
tissues and enlarge the damage to tumours, which has achieved good results. ${ }^{18-21}$ But the optimal management of craniopharyngioma remains controversial. In view of the lack of comprehensive evaluation on the efficacy and safety of PT for craniopharyngioma in adults, this study will comprehensively evaluate the tumour recurrence rate, median overall survival, progression-free survival (PFS) and treatment-related toxicity of PT for craniopharyngioma in adults through systematic review and meta-analysis, so as to provide clinical basis for PT.

\section{Objective}

Our study will combine evidence/data from existing randomised controlled trials (RCTs) to determine whether PT is safe and effective in the treatment of adults with craniopharyngioma.

\section{METHODS AND ANALYSIS}

This study will be written strictly according to the Preferred Reporting Items for Systematic Review and Meta-Analysis Protocols guidelines. ${ }^{22}$

\section{Inclusion and exclusion criteria}

Types of study

Studies to be included should meet the following criteria: (1) parallel-group RCTs; (2) objective to evaluate the efficacy and safety of PT for craniopharyngioma in adults; (3) studies with mixed population (ie, including both adults and children) will be included in our study if the subgroup analysis of adults and children is carried out, otherwise it will be excluded; (4) studies of patients who have received postoperative chemotherapy and previous RT will also be excluded, as this may affect our conclusions; (5) in addition, we will exclude studies that do not describe our outcome indicators such as the tumour recurrence rate, median overall survival, PFS and treatment-related toxicity; (6) there will be no restriction on language or publication status; (7) repeated studies will retain the latest one.

\section{Types of participants}

The target population of this study is adults aged 18 or above at the time of diagnosis, who were pathologically confirmed to be free from serious postoperative complications, such as severe pulmonary infection, stroke and other complications that could affect survival time. The same cohort of patients were reported in more than one study, and we will keep the latest one. There will be no restriction of race, nationality and source of participants.

\section{Intervention}

Our intervention is PT as a treatment for craniopharyngioma in adults.

\section{Comparator}

Our comparator is conventional X-ray beam RT as a treatment for craniopharyngioma in adults.

\section{Outcome}

Primary outcome

Our primary outcome, tumour recurrence and survival, will be used to assess the safety and efficacy of PT for craniopharyngioma in adults.

Evaluation indicators include:

1. Tumour recurrence rate.

2. Median overall survival.

3. PFS.

\section{Secondary outcome}

Our secondary outcome is an assessment of treatmentrelated toxicity (endocrine disorders, cognitive impairment, optic nerve injury and development of the second cancer).

\section{Study design}

Parallel-group RCTs of PT for craniopharyngioma in adults will be included.

\section{Information sources}

We will search six databases (MEDLINE, EMBASE, Web of Science, the Cochrane Library, Amed, Scopus), clinical research registration websites (WHO ICTRP and ISRCTN Register) and grey literature (the Health Management Information Database, OpenSIGLE Database and the National Technical Information Service), aiming to identify RCTs on PT for craniopharyngioma in adults between 1 January 1954 and 28 September 2021.

\section{Search strategy}

Two researchers (KZ and JY) formulated the search strategy, and the search strategy was based on the keywords of this study (PT and craniopharyngioma). Table 1 shows the search strategy for MEDLINE, and other database search strategies were adjusted slightly. Our search

\begin{tabular}{|c|c|}
\hline No & Search items \\
\hline 1. & Craniopharyngioma/ \\
\hline 2. & $\begin{array}{l}\text { (Craniopharyngioma* or craniopharyngeoma* } \\
\text { or pharyngioma* or pharyngeoma* or } \\
\text { "craniopharyngioma*" or "craniopharyngeoma*"). } \\
\text { ti,ab,cl,oa,kw. }\end{array}$ \\
\hline 3. & $\begin{array}{l}\text { ((Rathke* or "craniopharyngeal duct" or } \\
\text { "dysodontogenic epithelial" or "hypophyseal } \\
\text { duct"” or "third ventricle") adj3 (tumo?r* or } \\
\text { adenoma* or microadenoma* or neoplasm or } \\
\text { lesion*)).ti,ab,cl,oa,kw. }\end{array}$ \\
\hline 4. & Or/1-3 \\
\hline 5. & $\begin{array}{l}\text { (Proton }{ }^{*} \text { or proton beam }{ }^{*} \text { or new radiation }{ }^{*} \text { or PT } \\
\text { or PBT.ti,ab, } \mathrm{cl}, \mathrm{oa}, \mathrm{kw} \text {. }\end{array}$ \\
\hline 6. & 4 and 5 \\
\hline 7. & Limit 6 to adult \\
\hline 8. & Remove duplicates from 7 \\
\hline
\end{tabular}


strategy will not be restricted by ethnicity of the target population or language of publication.

\section{Study records}

Data management and selection process

We will import all the retrieved studies into EndNote software (V.X9). The selection process will be divided into two stages. In the first stage, two researchers (JW and HW) will independently read titles and abstracts in the software and classify all studies into three categories of 'Yes', 'No' and 'Uncertainty' based on our inclusion and exclusion criteria, excluding 'No'. In the second stage, two researchers (SL and KZ) will independently read the full text of 'Yes' and 'Uncertainty' and exclude inappropriate studies. The reasons for exclusion will be recorded in detail. If there is any controversy during this period, the decision will be discussed and made by four researchers (JW, HW, SL, KZ) together.

\section{Data collection process}

Two researchers (KZ and JY) will independently collect the data included in the study. Data collection will be divided into four parts. In the first part, the characteristics of the study include study author, publication date, journal name and country. In the second part, the characteristics of participants include gender, age and type of operation. The third part includes intervention and control information, the more detailed the better. The fourth part includes outcome and follow-up information. The third researcher (HW) will be responsible for examining the information collected and, if necessary, contact the author of the study newsletter for further information.

\section{Risk of bias}

Two researchers (PL and TZ) will use the Cochrane tool for assessing risk of bias in randomised trials-2 to evaluate the quality of included RCTs, which is divided into five domains of bias (bias from the randomisation process, bias due to deviations from intensified interevents, bias due to missing outcome data, bias in measurement of the outcome, bias in selection of the reported result).$^{23} \mathrm{In}$ each domain, a series of questions (signalling questions) need to be answered to obtain information on characteristics relevant to the study and a risk of bias calculated for each domain to ultimately obtain the full text of the risk of bias judgement (low risk of bias, some concerns, high risk of bias). If there is any dispute, the third researcher (AA) will participate in the discussion and settlement.

\section{Measures of treatment effect}

We will use RevMan V.5.2 for data synthesis and analysis. Dichotomous data will be analysed using a risk ratio with 95\% CIs. For continuous outcome data, the mean difference with $95 \%$ CIs will be used for analysis.

\section{Assessment of heterogeneity}

We will use the Mantel-Haenszel $\chi^{2}$ test and $\mathrm{I}^{2}$ statistic of homogeneity by RevMan V.5.2. According to the Cochrane Handbook, $I^{2}$ value can be used to evaluate heterogeneity.
0\%-40\%: might not be important; 30\%-60\%: may represent moderate heterogeneity; 50\%-90\%: may represent substantial heterogeneity; $75 \%-100 \%$ : considerable heterogeneity. ${ }^{24}$ When $\mathrm{p}<0.10$ and $\mathrm{I}^{2}>50 \%$, there is obvious heterogeneity. $\mathrm{I}^{2}>50 \%$ and $\mathrm{p}<0.10$ showed significant statistical heterogeneity.

\section{Data synthesis}

We will combine three or more studies for meta-analysis using random effects model. Heterogeneity will be judged by $\mathrm{I}^{2}$. If meta-analysis is not feasible due to clinical and methodological heterogeneity, the results will be summarised qualitatively.

\section{Assessment of reporting biases}

When the number of included studies reaches 10 or more, we will create a funnel plot of the outcome and evaluate whether there is a reporting bias based on its symmetry.

\section{Subgroup analysis}

We will conduct the following subgroup analyses to assess potential heterogeneity:

1. The classification of craniopharyngioma (eg, adamantinomatous craniopharyngioma and papillary craniopharyngioma)..$^{25} 26$

2. The location of craniopharyngioma (eg, according to the QST classification based on the origin of the tumor and the concept of membrane) ${ }^{27}$

3. The tumour size of craniopharyngioma (eg, greater than $4 \mathrm{~cm}$ and less than $4 \mathrm{~cm}){ }^{28}$

\section{Sensitivity analysis}

We will conduct a sensitivity analysis to assess the reliability of the meta-analysis by excluding studies with high risk of bias or missing reported data.

\section{Patient and public involvement}

No patient is involved in either the design or planning phase of this study.

\section{Ethics and dissemination}

Our study does not involve individual patients, so there is no need for ethical approval. The results of this study will be published in peer-reviewed journals or related conferences to evaluate the efficacy and safety of PT for craniopharyngioma in adults.

\section{DISCUSSION}

PT is an advanced RT method with less toxicity and better therapeutic effect than traditional X-ray RT. ${ }^{29}$ At present, PT has become an international focus and emphasis on tumour RT technology. The medical application of proton beam was first proposed by Wilson in $1946 .{ }^{30}$ In 1954, Tobias and others carried out the first PT in the world at Lawrence Berkeley National Laboratory at the University of California, and since then, Sweden, the former Soviet Union, and other countries have successively carried out clinical studies on PT. According to Particle Therapy Co-Operative Group, nearly 100 medical 
institutions around the world are currently using proton and heavy ion RT technology to implement tumour treatment, and there are more than 100 proton RT centres under construction, and nearly 200000 patients have received proton RT worldwide. ${ }^{31}$

The main techniques of PT for tumour treatment are active scanning and passive scattering. The Bragg peak of proton is obtained by modulator and collimator. At present, the common treatment methods of proton used in clinical tumour RT in the world are as follows: (1) proton point scan irradiation, to achieve precise control of tumour, but the operation process is more complex; (2) proton stereotactic RT, the main treatments of which are target area high-dose irradiation and fractional irradiation, with a limited clinical application; (3) intensity-modulated proton therapy (IMPT), realises dose intensity modulation and optimises dose distribution, especially the application of pencil beam scanning in IMPT, which avoids or reduces the radiation dose to surrounding normal tissues. In the treatment of head and neck tumours, PT can increase the radiation dose of the target area and reduce the toxicity of organs. ${ }^{32-34}$

The new version of the 2018 National Comprehensive Cancer Network guidelines takes PT as one of the standard treatments for head and neck cancer. ${ }^{35}$ A European centre study of 18 patients with craniopharyngioma who received PT found that one patient progressed 8.7 months after PT and then underwent surgery. Of the remaining patients at 18.4 months, five had complete remission, four had partial remission, seven had stable disease and there was no serious treatment-related toxicity during the treatment. ${ }^{17}$ Weber $e t$ $a l$ included 16 patients with craniopharyngioma, and finally analysed 15 patients, showing a 3-year overall survival rate of $75 \%$. While PT has similar efficacy to conventional X-ray RT, it is more protective to the temporal lobe and hippocampal formation. ${ }^{36}$

At present, there is a lack of comprehensive review on the efficacy and safety of PT for adults with craniopharyngioma; therefore, our study will comprehensively evaluate the recurrence rate, survival and treatment-related toxicity of PT for adults with craniopharyngioma through a systematic review and meta-analysis to provide a clinical basis for PT.

Contributors PL conceived the original idea for this systematic review. PL and JW drafted the manuscript. AA, KZ, JY, HW, SL and TZ revised the manuscript. All authors read and approved the final manuscript.

Funding This work was supported by special programme for young medical science and technology professionals in healthcare of the autonomous region of China (approval number: WJWY-202147).

Competing interests None declared.

Patient and public involvement Patients and/or the public were not involved in the design, or conduct, or reporting, or dissemination plans of this research.

Patient consent for publication Not required.

Provenance and peer review Not commissioned; externally peer reviewed.

Open access This is an open access article distributed in accordance with the Creative Commons Attribution Non Commercial (CC BY-NC 4.0) license, which permits others to distribute, remix, adapt, build upon this work non-commercially, and license their derivative works on different terms, provided the original work is properly cited, appropriate credit is given, any changes made indicated, and the use is non-commercial. See: http://creativecommons.org/licenses/by-nc/4.0/.
ORCID iDs

Tingrong Zhang http://orcid.org/0000-0002-3493-7403

Shaoshan Li http://orcid.org/0000-0002-9611-1244

\section{REFERENCES}

1 Bunin GR, Surawicz TS, Witman PA, et al. The descriptive epidemiology of craniopharyngioma. Neurosurg Focus 1997;3:e1

2 Wang G, Zhang X, Feng M, et al. Comparing survival outcomes of gross total resection and subtotal resection with radiotherapy for craniopharyngioma: a meta-analysis. J Surg Res 2018;226:131-9.

3 Cheng J, Fan Y, Cen B. Effect of preserving the pituitary stalk during resection of craniopharyngioma in children on the diabetes insipidus and relapse rates and long-term outcomes. J Craniofac Surg 2017;28:e591-5.

4 Rao YJ, Hassanzadeh C, Fischer-Valuck B, et al. Patterns of care and treatment outcomes of patients with craniopharyngioma in the National cancer database. J Neurooncol 2017;132:109-17.

5 Lee MH, Kim S-H, Seoul HJ, et al. Impact of maximal safe resection on the clinical outcome of adults with craniopharyngiomas. J Clin Neurosci 2012;19:1005-8.

6 Harrabi SB, Adeberg S, Welzel T, et al. Long term results after fractionated stereotactic radiotherapy (FSRT) in patients with craniopharyngioma: maximal tumor control with minimal side effects. Radiat Oncol 2014;9:203.

7 Lo AC, Howard AF, Nichol A, et al. Long-Term outcomes and complications in patients with craniopharyngioma: the British Columbia cancer agency experience. Int J Radiat Oncol Biol Phys 2014:88:1011-8.

8 Clark AJ, Cage TA, Aranda D, et al. A systematic review of the results of surgery and radiotherapy on tumor control for pediatric craniopharyngioma. Childs Nerv Syst 2013;29:231-8.

9 Zhang C, Verma V, Lyden ER, et al. The role of definitive radiotherapy in craniopharyngioma: a seer analysis. Am J Clin Oncol 2018;41:807-12.

10 Tringale KR, Nguyen TT, Karunamuni R, et al. Quantitative imaging biomarkers of damage to critical memory regions are associated with Post-radiation therapy memory performance in brain tumor patients. Int J Radiat Oncol Biol Phys 2019;105:773-83.

11 Parhar PK, Duckworth T, Shah P, et al. Decreasing temporal lobe dose with five-field intensity-modulated radiotherapy for treatment of pituitary macroadenomas. Int J Radiat Oncol Biol Phys 2010;78:379-84.

12 Albano L, Losa M, Flickinger J, et al. Radiotherapy of Parasellar tumours. Neuroendocrinology 2020;110:848-58.

13 Ajithkumar T, Mazhari A-L, Stickan-Verfürth M, et al. Proton Therapy for Craniopharyngioma - An Early Report from a Single European Centre. Clin Oncol 2018;30:307-16.

14 Bishop AJ, Greenfield B, Mahajan A, et al. Proton beam therapy versus conformal photon radiation therapy for childhood craniopharyngioma: multi-institutional analysis of outcomes, cyst dynamics, and toxicity. Int J Radiat Oncol Biol Phys 2014;90:354-61.

15 Tran S, Lim PS, Bojaxhiu B, et al. Clinical outcomes and quality of life in children and adolescents with primary brain tumors treated with pencil beam scanning proton therapy. Pediatr Blood Cancer 2020;67:e28465.

16 Marcus HJ, Rasul FT, Hussein Z, et al. Craniopharyngioma in children: trends from a third consecutive single-center cohort study. J Neurosurg Pediatr 2019:1-9.

17 Beltran C, Roca M, Merchant TE. On the benefits and risks of proton therapy in pediatric craniopharyngioma. Int $J$ Radiat Oncol Biol Phys 2012;82:e281-7.

18 Hussein Z, Glynn N, Martin N, et al. Temporal trends in craniopharyngioma management and long-term endocrine outcomes: a multicentre cross-sectional study. Clin Endocrinol 2021;94:242-9.

19 Enayet AER, Atteya MME, Taha $\mathrm{H}$, et al. Management of pediatric craniopharyngioma: 10-year experience from high-flow center. Childs Nerv Syst 2021;37:391-401.

20 Cossu G, Jouanneau E, Cavallo LM, et al. Surgical management of craniopharyngiomas in adult patients: a systematic review and consensus statement on behalf of the EANS skull base section. Acta Neurochir 2020;162:1159-77.

21 Park HJ, Dho Y-S, Kim JH, et al. Recurrence rate and prognostic factors for the adult craniopharyngiomas in long-term follow-up. World Neurosurg 2020;133:e211-7.

22 Shamseer L, Moher D, Clarke M, et al. Preferred reporting items for systematic review and meta-analysis protocols (PRISMA-P) 2015 elaboration and explanation. BMJ 2015;350:g7647. 
23 Sterne JAC, Savović J, Page MJ, et al. Rob 2: a revised tool for assessing risk of bias in randomised trials. BMJ 2019;366:14898.

24 Higgins JPT, Thomas J, Chandler J, eds. Cochrane Handbook for Systematic Reviews of Interventions version 6.2 (updated February 2021). Cochrane, 2021.

25 Müller HL, Merchant TE, Warmuth-Metz M, et al. Craniopharyngioma. Nat Rev Dis Primers 2019;5:75.

26 Winkfield KM, Linsenmeier C, Yock TI, et al. Surveillance of craniopharyngioma cyst growth in children treated with proton radiotherapy. Int J Radiat Oncol Biol Phys 2009;73:716-21.

27 Fan J, Liu Y, Pan J, et al. Endoscopic endonasal versus transcranial surgery for primary resection of craniopharyngiomas based on a new QST classification system: a comparative series of 315 patients. J Neurosurg 2021:1-12.

28 Sadhasivam S, Menon G, Abraham M, et al. The implication of giant tumor size on surgical resection, oncological, and functional outcomes in craniopharyngioma. Pituitary 2020;23:515-25.

29 Sakurai H, Ishikawa H, Okumura T. Proton beam therapy in Japan: current and future status. Jpn J Clin Oncol 2016;46:885-92.
30 WILSON RR. Radiological use of fast protons. Radiology 1946;47:487-91.

31 Lawrence JH, Tobias CA, Born JL. Pituitary irradiation with highenergy proton beams a preliminary report. $J$ Cancer Research 1958;18:121-34.

32 Cotter SE, McBride SM, Yock TI. Proton radiotherapy for solid tumors of childhood. Technol Cancer Res Treat 2012;11:267-78.

33 Weber DC, Lomax AJ, Rutz HP, et al. Spot-scanning proton radiation therapy for recurrent, residual or untreated intracranial meningiomas. Radiother Oncol 2004;71:251-8.

34 Frank SJ, Cox JD, Gillin M, et al. Multifield optimization intensity modulated proton therapy for head and neck tumors: a translation to practice. Int J Radiat Oncol Biol Phys 2014;89:846-53.

35 Colevas AD, Yom SS, Pfister DG, et al. NCCN guidelines insights: head and neck cancers, version 1.2018. J Natl Compr Canc Netw 2018;16:479-90.

36 Weber DC, Ares C, Albertini F, et al. Pencil beam scanning proton therapy for pediatric Parameningeal rhabdomyosarcomas: clinical outcome of patients treated at the Paul Scherrer Institute. Pediatr Blood Cancer 2016;63:1731-6. 